\title{
Combined Load Diagram for a Wind Tunnel Strain-Gage Balance
}

\author{
N. Ulbrich* \\ Jacobs Technology Inc., Moffett Field, California 94035-1000
}

\begin{abstract}
Combined Load Diagrams for Direct-Read, Force, and Moment Balances are discussed in great detail in the paper. The diagrams, if compared with a corresponding combined load plot of a balance calibration data set, may be used to visualize and interpret basic relationships between the applied balance calibration loads and the load components at the forward and aft gage of a strain-gage balance. Lines of constant total force and moment are identified in the diagrams. In addition, the lines of pure force and pure moment are highlighted. Finally, lines of constant moment arm are depicted. It is also demonstrated that each quadrant of a Combined Load Diagram has specific regions where the applied total calibration force is at, between, or outside of the balance gage locations. Data from the manual calibration of a Force Balance is used to illustrate the application of a Combined Load Diagram to a realistic data set.
\end{abstract}

\section{Nomenclature}

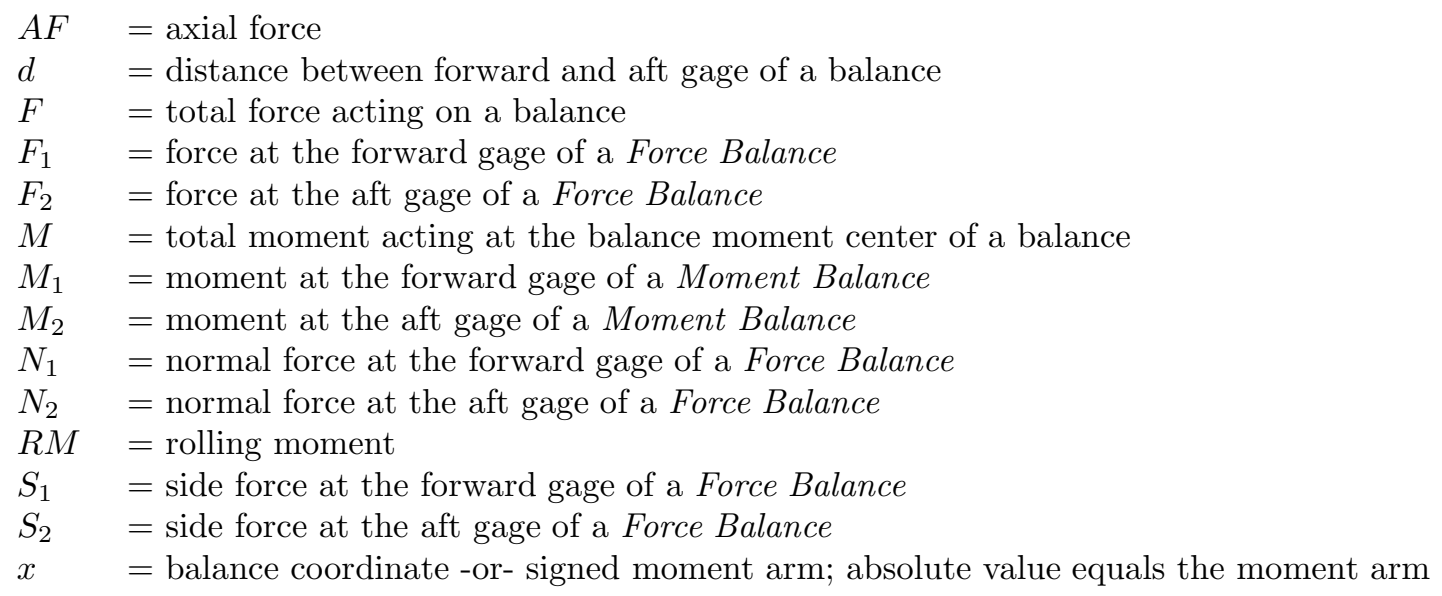

\section{Introduction}

During design and evaluation of a load schedule for the calibration of a wind tunnel strain-gage balance it is often useful to highlight the relationship between magnitude \& location of the applied calibration loads in a combined load plot of the calibration data. An analysis of this plot may lead to a better use of existing calibration hardware as the connection between the applied loads and the chosen moment arms can directly be seen in a single plot. In addition, the calibration load schedule may better be tailored to the expected use envelope of the balance during a wind tunnel test which may result in a more accurate regression model of the balance calibration data.

\footnotetext{
* Aerodynamicist; Jacobs Technology Inc. Copyright ( $(2010$ by the American Institute of Aeronautics and Astronautics, Inc. - The U.S. Government has a royalty-free license to exercise all rights under the copyright claimed herein for Governmental purposes. All other rights are reserved by the copyright owner.
} 
A specific set of generic diagrams are used at NASA Ames' Balance Calibration Lab for the visualization and interpretation of the relationship between magnitude \& location of the applied calibration loads in a combined load plot. They are called Combined Load Diagrams. Different Combined Load Diagrams are defined for the different types of balances that are used in the aerospace testing community today (see Ref. [1] for a description of the different types). For a Direct-Read Balance, for example, the Combined Load Diagram looks at the calibration loads by plotting the total moment at the balance moment center versus the total force. For a Force or Moment Balance, on the other hand, the diagram looks at the plot of the force (or moment) at the aft gage versus the force (or moment) at the forward gage.

Certain lines and regions are highlighted in the Combined Load Diagram that make it easier to interpret the calibration data in a combined load plot. The lines of constant total force and moment are marked. This also includes the lines of pure force and pure moment. In addition, the lines of constant moment arm are displayed. Finally, regions in the Combined Load Diagram are identified that help recognize (i) the sign of the applied calibration forces and moments and (ii) the location of the applied calibration forces relative to the location of the balance gages. It is recommended to compare the generic Combined Load Diagram of the given balance type (i.e., Direct-Read, Force, or Moment Balance) with the corresponding combined load plot of the calibration data in order to gain a better understanding of the depicted load schedule.

In the next section of the paper the Combined Load Diagrams for a Direct-Read, Force, and Moment Balance are discussed in more detail. In addition, data from the manual calibration of a Force Balance will be used to illustrate the application of a combined load diagram to a realistic balance calibration data set.

\section{Combined Load Diagram}

\section{A. Direct-Read Balance}

Figure 1a shows the Combined Load Diagram for a Direct-Read Balance. Detailed derivations of the lines of constant force, moment, and moment arm are given in Appendix 1 of the paper. Lines of constant total force and moment are depicted. The lines of constant total force are lines that are perpendicular to the abscissa of the coordinate system. The lines of constant total moment, on the other hand, are lines that are parallel to the abscissa of the coordinate system. The pure force line $(M=0)$ is the abscissa of the coordinate system. The pure moment line $(F=0)$ is the ordinate of the coordinate system. The pure moment line also represents the hypothetical situation when the magnitude of the moment arm $|x|$ approaches infinity. Therefore, a pure moment can only be applied to the balance by using a force couple.

The lines of constant moment arm can also be seen Fig. 1a. All lines of constant moment arm must go through the origin of the coordinate system. The abscissa is identical with the line for a moment arm of zero. In this case, the total force is at the balance moment center. The ordinate equals the line for a moment arm of infinite magnitude. The two diagonal lines represent the situation when the calibration force is exactly at the gages $(x= \pm d / 2)$.

Figure 1b shows quadrant characteristics of the Combined Load Diagram for a Direct-Read Balance. The color yellow marks regions where the total calibration force is applied at or between the forward and aft gage of the balance. The color orange marks regions where the total calibration force is applied outside of the location of the gages. The dividing lines between the yellow and orange regions are the two diagonal lines that represent the situation when the calibration force is exactly at the gages $(x= \pm d / 2)$.

\section{B. Force Balance}

Figure 2a shows the Combined Load Diagram for a Force Balance. Detailed derivations of the lines of constant force, moment, and moment arm are given in Appendix 2 of the paper. Lines of constant total force and moment are depicted. The location of a line of constant load depends on (i) the magnitude of the calibration force, (ii) the sign of the calibration force, and (iii) the moment arm of the force relative to the balance moment center. The slope of the lines in Fig. 2a is \pm 1 because it is assumed that the balance moment center is located halfway between the forward and aft gage.

It is interesting to note that both the pure moment line $(F=0)$ and the pure force line $(M=0)$ in Fig. 2a are the principle diagonals of the four quadrants of the Combined Load Diagram. The pure force line is the principle diagonal in the first and third quadrant. The pure moment line, on the other hand, is the principle diagonal in the second and fourth quadrant. Again, similar to the situation for a Direct Read 
Balance, the pure moment line represents the hypothetical situation when the magnitude of the moment arm approaches infinity. Therefore, a pure moment can physically only be applied to the balance if a force couple is applied.

The lines of constant moment arm can also be seen Fig. 2a. All lines of constant moment arm must go through the origin of the coordinate system. The abscissa is identical with the line for a moment arm of $x=+d / 2$. The ordinate is identical with the line for a moment arm of $x=-d / 2$. The principle diagonal of the first and third quadrant is identical with the line for a moment arm of zero. In this case, the total calibration force is applied at the balance moment center (coordinate $x=0$ ). The principle diagonal of the second and fourth quadrant, on the other hand, is identical with the line for a moment arm of infinity.

Figure 2b shows characteristics of each quadrant of the Combined Load Diagram for a Force Balance. The first and third quadrant (yellow color) contain only those points where the calibration force is located between the gages. The second and fourth quadrant, on the other hand, contain points where the calibration force is located outside of the gages.

\section{Moment Balance}

Figure 3a shows the Combined Load Diagram for a Moment Balance. Detailed derivations of the lines of constant force, moment, and moment arm are given in Appendix 3 of the paper. Lines of constant total force and moment are depicted. The location of a line of constant load depends on (i) the magnitude of the calibration force, (ii) the sign of the calibration force, and (iii) the moment arm of the force relative to the balance moment center. The slope of the lines in Fig. 3a is \pm 1 because it is assumed that the balance moment center is located halfway between the forward and aft gage.

Again, similar to the Force Balance, the pure moment line $(F=0)$ and the pure force line $(M=0)$ in Fig. 3a are the principle diagonals of the four quadrants of the Combined Load Diagram. This time, however, the pure force line is the principle diagonal in the second and fourth quadrant. The pure moment line, on the other hand, is the principle diagonal in the first and third quadrant. Again, similar to the situation for a Direct Read Balance, the pure moment line represents the hypothetical situation when the magnitude of the moment arm approaches infinity. Therefore, a pure moment can physically only be applied to the balance if a force couple is applied.

The lines of constant moment arm can also be seen Fig. 3a. All lines of constant moment arm must go through the origin of the coordinate system. The abscissa is identical with the line for a moment arm of $x=-d / 2$. The ordinate is identical with the line for a moment arm of $x=+d / 2$. The principle diagonal of the second and fourth quadrant is identical with the line for a moment arm of zero. In this case, the total calibration force is applied at the balance moment center (coordinate $x=0$ ). The principle diagonal of the first and third quadrant, on the other hand, is identical with the line for a moment arm of infinity.

Figure 3b shows characteristics of each quadrant of the Combined Load Diagram for a Moment Balance. The second and fourth quadrant (yellow color) contain only those points where the calibration force is located between the gages. The first and third quadrant, on the other hand, contain points where the calibration force is located outside of the gages.

Comparing the Combined Load Diagram for a Moment Balance (Figs. 3a and 3b) with the Combined Load Diagram for a Force Balance (Figs. 2a and 2b) we see that the lines of constant total force, constant total moment, and constant moment arm of the Moment Balance can be obtained from the corresponding lines of the Force Balance by using a simple $-90^{\circ}$ rotation about the origin of the coordinate system.

\section{Discussion of Example}

Data from a simple manual calibration of the Ames MK-III-C balance may be used to illustrate the application of the Combined Load Diagram to a realistic calibration data set. The balance is a six-component Force Balance (Task). It measures five forces and one moment $\left(N_{1}, N_{2}, S_{1}, S_{2}, R M, A F\right)$. It has a diameter of 2.0 inches and a total length of 11.25 inches. For the present example it was decided to focus on the loads in the $N_{1}-N_{2}$ plane. Table 1 below shows the capacity of each load component.

The balance calibration was performed using the "hand load" method. A total of 247 data points were taken in 13 load series. Combined loadings were only applied to the normal force components $\left(N_{1}, N_{2}\right)$.

Figure 4 shows the combined load plot for the $N_{1}-N_{2}$ force combination. Five distinct lines can be seen

American Institute of Aeronautics and Astronautics 
in the combined load plot of the selected load combination. Figure 2a, i.e., the Combined Load Diagram for a Force Balance, may be used to interpret these lines.

Table 1: Load capacities of MK-III-C balance.

\begin{tabular}{|l|c|c|c|c|c|c|}
\hline & $N_{1}, \mathrm{lbs}$ & $N_{2}, \mathrm{lbs}$ & $S_{1}, \mathrm{lbs}$ & $S_{2}, \mathrm{lbs}$ & $R M$, in-lbs & $A F, \mathrm{lbs}$ \\
\hline CAPACITY & 900 & 900 & 450 & 450 & 1200 & 500 \\
\hline
\end{tabular}

Points on the abscissa (line 1) have a $N_{2}$ load component of zero. Therefore, these points represent loadings that were applied at the forward gage of the balance (coordinate $x=+3.625[\mathrm{in}]$ ). Points on the ordinate (line 2), on the other hand, have a $N_{1}$ component of zero. Therefore, points on the ordinate represent loadings that were applied at the aft gage of the balance (coordinate $x=-3.625[\mathrm{in}]$ ).

Comparing Fig. 2b with Fig. 4 we conclude that the calibration points located inside of the second and fourth quadrant (line 3) must have been taken outside of the gage locations. This observation can be confirmed if the load transformations given in Ref. [2] are applied to estimate the moment arm. The calculated coordinate (in this case the moment arm) of the applied calibration force is determined to be $x=+10.375[\mathrm{in}]$. Therefore, the calibration force was located $6.750[\mathrm{in}]$ forward of the forward gage.

The principle diagonal of the second and fourth quadrant (line 4) is the pure moment line. In theory, calibration points can only be placed on this line if a force couple is applied during the calibration. It would have been possible to better approximate the influence of a pure moment on the gage outputs if calibration points would have been obtained for a signed moment arm of $x=-10.375[\mathrm{in}]$. In that case, calibration points would have appeared on line 5 and points on both line 3 and line 5 could have been used for the regression analysis of the calibration data. Consequently, a very good approximation of the influence of a pure moment on the gage outputs could have been included in the regression model of the calibration data as the pure moment line (line 4 ) is located halfway between line 3 and line 5.

\section{Summary and Conclusions}

The Combined Load Diagrams for a Direct-Read, Force, and Moment Balance were derived and discussed in great detail. The diagrams may be used to analyze and interpret the combined load plots of three different balance types. Results of this analysis may help better understand the load schedule design that was applied during the calibration of a balance. In addition, the diagrams may be used to quickly understand how to get calibration points in certain regions of the combined load plot that may be needed in order to develop a more reliable regression model of the balance behavior. A combined load plot from the calibration of a six-component Force Balance was used to illustrate benefits of the diagrams.

Often, because of calibration hardware limitations, it is difficult to apply a pure moment during the calibration of a balance. These difficulties may be avoided if the influence of a pure moment loading on the gage outputs is approximated by combining a large "signed" moment arm (e.g., $x= \pm 3 d$ ) and a total force such that the design load limit of the total moment of the balance is not exceeded. Then, calibration points are obtained that are much closer to the pure moment condition than those calibration points that are traditionally applied at or between the forward and aft gage of the balance. Consequently, the final regression model of the calibration data may better be able to predict pure moment loadings during a wind tunnel test even though they were not directly applied during the calibration.

\section{Acknowledgements}

The author would like to thank Tom Bridge and Tom Volden of Jacobs Technology for their constructive review of the final manuscript. The work reported in this paper was partially supported by NASA's Aeronautic Test Program and the Wind Tunnel Division at Ames Research Center under contract NNA09DB39C.

\section{References}

${ }^{1}$ AIAA/GTTC Internal Balance Technology Working Group, "Recommended Practice, Calibration and 
Use of Internal Strain-Gage Balances with Application to Wind Tunnel Testing," AIAA R-091-2003, published by the American Institute of Aeronautics and Astronautics, Reston, Virginia, 2003, pp. 4-5.

${ }^{2}$ Ulbrich, N. and Bader, J., "Analysis of Sting Balance Calibration Data using Optimized Regression Models," AIAA 2009-5372, paper presented at the 45th AIAA/ASME/SAE/ASEE Joint Propulsion Conference \& Exhibit, Denver, Colorado, August 2009, pp. 18-21. 


\section{Appendix 1: Direct-Read Balance - Lines of Constant Load \& Moment Arm}

The interpretation and use of the Combined Load Diagram for a Direct-Read Balance needs information about the exact location of the lines of (i) constant total force, (ii) constant total moment, and (iii) constant moment arm. The location of these lines can be obtained after analyzing the basic relationship between the total force, total moment, and moment arm in more detail (see Fig. 1a).

Figure 1 shows the total force and total moment that act at the balance moment center of a Direct-Read Balance (see Ref. [1] for a detailed description of the balance type). The basic relationship between total force $F$, total moment $M$, and "signed" moment arm $x$ is given as follows:

$$
M=M(F, x)=F \cdot x
$$

where $|x|$ equals the applied moment arm. The Combined Load Diagram of the balance is simply defined as the plot of the total moment $M$ at the balance moment center versus the total force $F$.

Constant Total Force: The lines of constant total force are vertical lines that are parallel to the ordinate of the Combined Load Diagram (see Fig. 1a). They can be described by the following relationship:

$$
F=\text { constant } \Longleftrightarrow \text { line of constant total force }
$$

The pure moment line, i.e., the line where the total force is zero, is the vertical line that goes through the origin of the Combined Load Diagram. It is described by the following equation:

$$
F=0 \Longleftrightarrow \quad \text { line of pure moment }
$$

The pure moment line is identical with the ordinate of the coordinate system of the combined load diagram of the Direct-Read Balance.

Constant Total Moment: The lines of constant total moment are horizontal lines that are parallel to the abscissa of the Combined Load Diagram (see Fig. 1a). They can be described using the following equation:

$$
M=\text { constant } \Longleftrightarrow \quad \text { line of constant total moment }
$$

The pure force line, i.e., the line where the total moment is zero, is the horizontal line that goes through the origin of the Combined Load Diagram. It is described by the following equation:

$$
M=0 \Longleftrightarrow \quad \text { line of pure force }
$$

The pure force line is identical with the abscissa of the coordinate system of the combined load diagram of the Direct-Read Balance.

Constant Moment Arm: The location of the lines of constant moment arm may simply be identified in the combined load diagram by using the basic relationship between the total force $F$, the total moment $M$, and the "signed" moment arm $x$. Therefore, assuming that the "signed" moment arm $x$ is kept constant, it is concluded from Eq. (1) that all lines of constant moment arm must go through the origin of the coordinate system as the total moment $M$ is directly proportional to the total force $F$. In addition, the partial derivative of the total moment $M$ with respect to the total force $F$ is given as:

$$
\frac{\partial M}{\partial F}=x
$$

The derivative equals the "signed" moment arm $x$. Therefore, it is concluded from Eq. (4) that the slope of a line of constant moment arm in the Combined Load Diagram of a Direct-Read Balance is constant. A positive slope in the Combined Load Diagram means that the "signed" constant moment arm is positive. A negative slope means that the "signed" constant moment arm is negative.

In the limit, assuming that the moment arm $|x|$ approaches $\infty$, the line of the constant moment arm will collapse with the ordinate of the Combined Load Diagram. The total force on the ordinate of the Combined Load Diagram is zero - in other words - the ordinate is the pure moment line. This conclusion has an important practical application. Often, it is difficult to directly apply a pure moment during the 
calibration of a balance. In that case special calibration hardware needs to be used and the exact alignment of the applied calibration loads becomes an issue. These difficulties may be avoided if, instead of applying a pure moment during the calibration, the impact of a pure moment loading on the balance gage outputs is approximated by combining a large "signed" moment arm $x$ (e.g., $x= \pm 3 d$ ) and a total force $F$ such that the design load limit of the total moment $M$ of the balance is not exceeded. Then, calibration points will be obtained for the final regression analysis of the calibration data that are closer to the pure moment condition than those calibration points that are traditionally applied at or between the forward and aft gage of the balance. 


\section{Appendix 2: Force Balance - Lines of Constant Load \& Moment Arm}

The interpretation and use of the Combined Load Diagram for a Force Balance needs information about the exact location of the lines of (i) constant total force, (ii) constant total moment, and (iii) constant moment arm. Figure 2a shows loads acting at the balance moment center and at the forward \& aft gages of a Force Balance. It is assumed that the balance moment center is located halfway between the forward and aft gage of the balance. The Combined Load Diagram is defined by plotting the force at the aft gage $\left(F_{2}\right)$ versus the force at the forward gage $\left(F_{1}\right)$.

The load transformation equations for a Force Balance may be used to identify the lines of constant total force, constant total moment, and constant moment arm in the Combined Load Diagram of a Force Balance. We know, using derivations presented in Ref. [2] and using Fig. 2a, that the total force and the total moment are given by the following equations:

$$
\begin{gathered}
F=F_{1}+F_{2} \\
M=\left[F_{1}-F_{2}\right] \cdot \frac{d}{2}
\end{gathered}
$$

Constant Total Force: The lines of constant total force $F$ in the combined load diagram of a Force Balance can be obtained from Eq. ( $5 a$ ). Solving, e.g., Eq. ( $5 a$ ) for $F_{2}$, we get:

$$
F_{2}=\underbrace{[F]}_{\text {constant }}-F_{1} \Longleftrightarrow \text { line of constant total force }
$$

The pure moment line is defined as the line where the total force $F$ is zero. Therefore, after setting the total force used in Eq. (6a) to zero, we get the equation of the pure moment line:

$$
F_{2}=-F_{1} \Longleftrightarrow \quad \text { line of pure moment }
$$

Constant Total Moment: The line of constant total moment still needs to be determined. Now, after solving Eq. $(5 b)$ for $F_{2}$, we get:

$$
F_{2}=\underbrace{\left[\frac{-2 \cdot M}{d}\right]}_{\text {constant }}+F_{1} \Longleftrightarrow \quad \text { line of constant total moment }
$$

The pure force line is defined as the line where the total moment $M$ is zero. Therefore, after setting the total moment in Eq. (7a) to zero, we get the equation of the pure force line:

$$
F_{2}=F_{1} \Longleftrightarrow \quad \text { line of pure force }
$$

Constant Moment Arm: The lines of constant moment arm in the Combined Load Diagram of a Force Balance may be obtained after replacing the total moment on the left hand side of Eq. (1) with the right hand side of Eq. (5b) and the total force on the right hand side of Eq. (1) with the right hand side of Eq. (5a). Then, we get:

$$
\left[F_{1}-F_{2}\right] \cdot \frac{d}{2}=\left[F_{1}+F_{2}\right] \cdot x
$$

where $x$ is the "signed" moment arm. Rearranging terms in Eq. (8) and solving for the force component $F_{2}$, we get:

$$
\left.F_{2}=F_{1} \cdot\left[\frac{d-2 \cdot x}{d+2 \cdot x}\right] \Longleftrightarrow \text { line of constant moment arm (version } 1\right)
$$

which may also be written as

$$
\left.F_{2}=F_{1} \cdot\left[\frac{d / x-2}{d / x+2}\right] \Longleftrightarrow \quad \text { line of constant moment arm (version } 2\right)
$$


Alternatively, Eq. (9a) may be solved for the force component $F_{1}$. Then, we get:

$$
\left.F_{1}=F_{2} \cdot\left[\frac{d+2 \cdot x}{d-2 \cdot x}\right] \Longleftrightarrow \text { line of constant moment arm (version } 3\right)
$$

It is concluded from Eqs. $(9 a)$ and $(9 b)$ that the lines of constant moment arm are straight lines that must go through the origin of the Combined Load Diagram as the fractions on the right hand side of the two equations satisfy the following conditions:

$$
x=[\text { first constant }] \quad \Longrightarrow\left[\frac{d-2 \cdot x}{d+2 \cdot x}\right]=[\text { second constant }] \quad \Longrightarrow \quad F_{2}=F_{1} \cdot[\text { second constant }]
$$

Similary, after taking a closer look at Eq. (9c), it can also be concluded that

$$
x=[\text { first constant }] \quad \Longrightarrow\left[\frac{d+2 \cdot x}{d-2 \cdot x}\right]=[\text { third constant }] \quad \Longrightarrow \quad F_{1}=F_{2} \cdot[\text { third constant }]
$$

Now, four limiting cases need to be evaluated in order to complete the investigation of the equations of the constant moment arm. We need to know what lines describe the following four moment arms: $x=+d / 2$, $x=-d / 2, x=0$, and $x \rightarrow \pm \infty$. Equations (9a), (9b), and (9c) may be applied to find the corresponding lines in the Combined Load Diagram. The first line is obtained after applying the condition $x=+d / 2$ to Eq. (9a). Then, we get:

$$
x=+d / 2 \quad \Longrightarrow \quad F_{2}=0
$$

In the next step, after applying the condition $x=-d / 2$ to Eq. (9c), we get the equation of the second line:

$$
x=-d / 2 \quad \Longrightarrow \quad F_{1}=0
$$

Similarly, after applying the condition $x=0$ to Eq. $(9 a)$, we get the equation of the third line:

$$
x=0 \quad \Longrightarrow \quad F_{2}=F_{1} \Longleftrightarrow \text { line of pure force }
$$

It is observed that the equation of the line for $x=0$, i.e., Eq. $(10 c)$, is identical with the equation of the pure force line that is given in Eq. (7b). This result is expected as the force is applied in this case at the balance moment center (see also Fig. 2a).

Finally, after taking the limit $x \rightarrow \pm \infty$ of the fraction given on the right hand side of Eq. (9b), we get the equation of the fourth line:

$$
\lim _{x \rightarrow \pm \infty}\left[\frac{d / x-2}{d / x+2}\right]=-1 \quad \Longrightarrow \quad F_{2}=-F_{1} \quad \Longleftrightarrow \quad \text { line of pure moment }
$$

It is interesting to note that the equation of the fourth line defined in Eq. (10d) is identical with the pure moment line that is given in Eq. (6b). Therefore, it is concluded that the gage outputs of the Force Balance will more and more resemble the gage outputs for a pure moment condition if the absolute value of the "signed" moment arm $x$ is getting significantly larger than the distance $d$ between the forward and aft gages of the balance. 


\section{Appendix 3: Moment Balance - Lines of Constant Load \& Moment Arm}

The interpretation and use of the Combined Load Diagram for a Moment Balance needs information about the exact location of the lines of (i) constant total force, (ii) constant total moment, and (iii) constant moment arm. Figure 3a shows the loads acting on a balance moment center and at the forward \& aft gages of a Moment Balance. It is assumed that the balance moment center is located halfway between the forward and aft gage of the balance. The Combined Load Diagram is defined by plotting the moment at the aft gage $\left(M_{2}\right)$ versus the moment at the forward gage $\left(M_{1}\right)$.

The classical load transformation equations for a Moment Balance may be used to identify the lines of constant total force, constant total moment, and constant moment arm in the Combined Load Diagram of a Moment Balance. We know, after reviewing the derivations given in Ref. [2] and after looking at Fig. 3a, that the total force and the total moment are given by the following equations:

$$
\begin{aligned}
& F=\left[M_{2}-M_{1}\right] \cdot \frac{1}{d} \\
& M=\left[M_{1}+M_{2}\right] \cdot \frac{1}{2}
\end{aligned}
$$

Constant Total Force: The lines of constant total force $F$ in the Combined Load Diagram of a Moment Balance can be obtained from Eq. (11a). Solving, e.g., Eq. (11a) for $M_{2}$, we get:

$$
M_{2}=\underbrace{[F \cdot d]}_{\text {constant }}+M_{1} \Longleftrightarrow \quad \text { line of constant total force }
$$

The pure moment line is defined as the line where the total force $F$ is zero. Therefore, after setting the total force in Eq. (12a) to zero, we get the equation of the pure moment line:

$$
F=0 \Longrightarrow M_{2}=M_{1} \Longleftrightarrow \quad \text { line of pure moment }
$$

Constant Total Moment: The line of constant total moment still needs to be determined. Now, after solving Eq. (11b) for $M_{2}$, we get:

$$
M_{2}=\underbrace{[2 \cdot M]}_{\text {constant }}-M_{1} \Longleftrightarrow \quad \text { line of constant total moment }
$$

The pure force line is defined as the line where the total moment $M$ is zero. Therefore, after setting the total moment in Eq. (13a) to zero, we get the equation of the pure force line:

$$
M=0 \Longrightarrow M_{2}=-M_{1} \Longleftrightarrow \quad \text { line of pure force }
$$

Constant Moment Arm: The lines of constant moment arm in the Combined Load Diagram of a Moment Balance may be obtained after replacing the total moment on the left hand side of Eq. (1) with the right hand side of Eq. (11b) and the total force on the right hand side of Eq. (1) with the right hand side of Eq. (11a). Then, we get:

$$
\left[M_{1}+M_{2}\right] \cdot \frac{1}{2}=\left[M_{2}-M_{1}\right] \cdot \frac{x}{d}
$$

Rearranging terms in Eq. (14), solving for the moment component $M_{2}$, and after simplifying terms, we get:

$$
\left.M_{2}=M_{1} \cdot\left[\frac{2 \cdot x+d}{2 \cdot x-d}\right] \Longleftrightarrow \quad \text { line of constant moment arm (version } 1\right)
$$

which may also be written as

$$
\left.M_{2}=M_{1} \cdot\left[\frac{2+d / x}{2-d / x}\right] \Longleftrightarrow \quad \text { line of constant moment arm (version } 2\right)
$$


Alternatively, Eq. (15a) may be solved for the moment component $M_{1}$. Then, we get:

$$
\left.M_{1}=M_{2} \cdot\left[\frac{2 \cdot x-d}{2 \cdot x+d}\right] \Longleftrightarrow \quad \text { line of constant moment arm (version } 3\right)
$$

It is concluded from Eqs. $(15 a)$ and $(15 b)$ that the lines of constant moment arm are straight lines that must go through the origin of the Combined Load Diagram as the fractions on the right hand side of the two equations satisfy the following conditions:

$x=[$ first constant $] \quad \Longrightarrow\left[\frac{2 \cdot x+d}{2 \cdot x-d}\right]=[$ second constant $] \quad \Longrightarrow \quad M_{2}=M_{1} \cdot[$ second constant $]$

Similary, after taking a closer look at Eq. (15c), it can also be concluded that

$$
x=[\text { first constant }] \quad \Longrightarrow\left[\frac{2 \cdot x-d}{2 \cdot x+d}\right]=[\text { third constant }] \quad \Longrightarrow \quad M_{1}=M_{2} \cdot[\text { third constant }]
$$

Now, four limiting cases need to be evaluated in order to complete the investigation of the lines for constant moment arm. We need to know which equations describe the lines of the following four moment arms: $x=+d / 2, x=-d / 2, x=0$, and $x \rightarrow \pm \infty$. Equations (15a), (15b), and (15c) may be applied to find the corresponding lines in the Combined Load Diagram. The equation of the first line is obtained after applying the condition $x=-d / 2$ to Eq. (15a). Then, we get:

$$
x=-d / 2 \quad \Longrightarrow \quad M_{2}=0
$$

Now, after applying the condition $x=+d / 2$ to Eq. (15c), we get the equation of the second line:

$$
x=+d / 2 \quad \Longrightarrow \quad M_{1}=0
$$

Similarly, after applying the condition $x=0$ to Eq. $(15 a)$, we get the equation of the third line:

$$
x=0 \Longrightarrow M_{2}=-M_{1} \Longleftrightarrow \quad \text { line of pure force }
$$

It is observed that the line for $x=0$, i.e., Eq. (16c), is identical with the pure force line that was derived in Eq. (13b). This observation is expected as the force is applied in this case at the balance moment center (see also Fig. 3a).

Finally, after taking the limit $x \rightarrow \pm \infty$ of the fraction given on the right hand side of Eq. (15b), we get the equation of the fourth line:

$$
\lim _{x \rightarrow \pm \infty}\left[\frac{2+d / x}{2-d / x}\right]=1 \quad \Longrightarrow \quad M_{2}=M_{1} \Longleftrightarrow \quad \text { line of pure moment }
$$

It is interesting to note that the equation of the fourth line given in Eq. (16d) is identical with the equation of the pure moment line that is defined in Eq. (12b). Therefore, it is concluded that the gage outputs of a Moment Balance will more and more resemble the gage outputs for a pure moment condition if the absolute value of the "signed" moment arm $x$ is significantly larger than the distance $d$ between the forward and aft gages of the balance. 

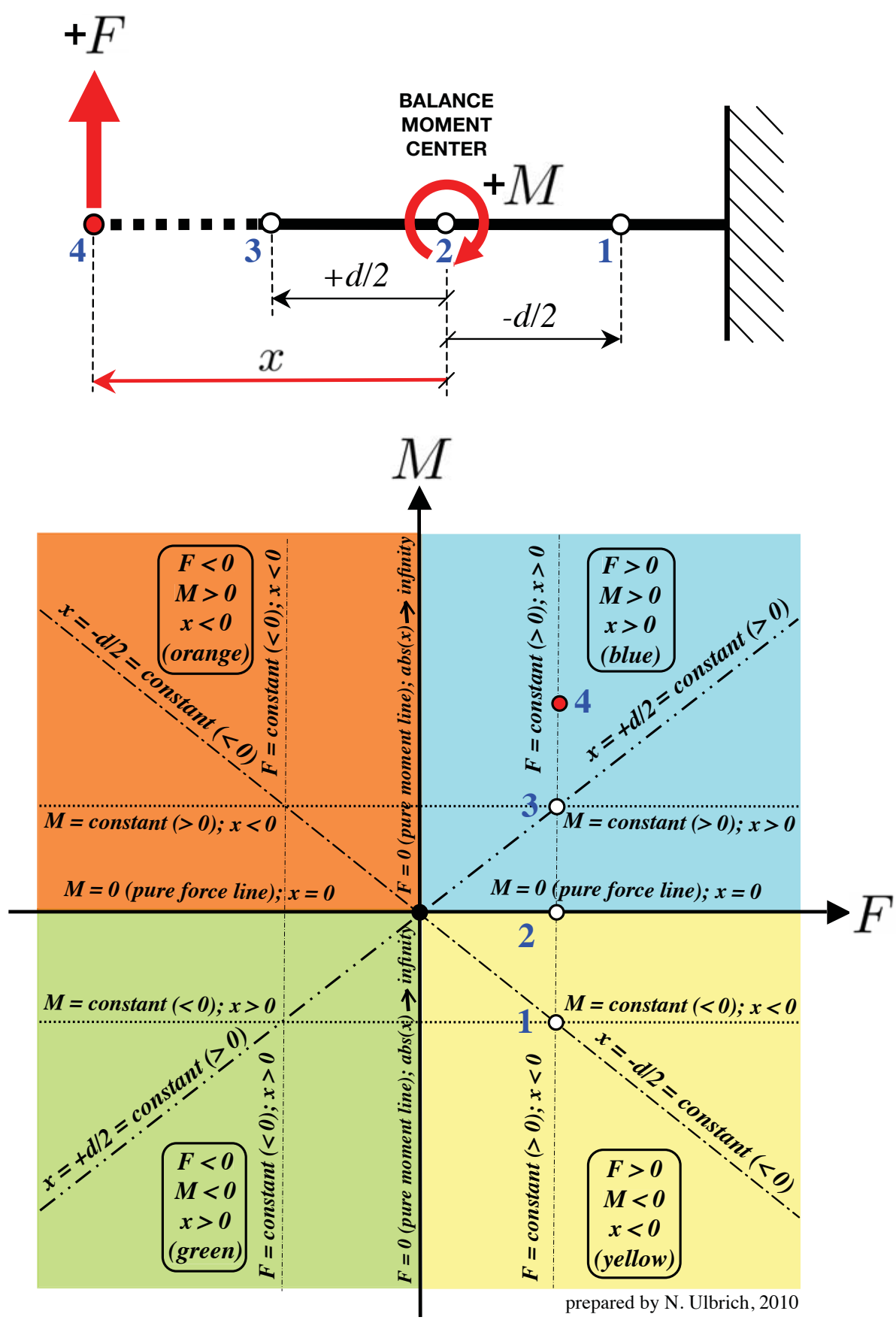

Fig. 1a Combined Load Diagram for a Direct-Read Balance. 


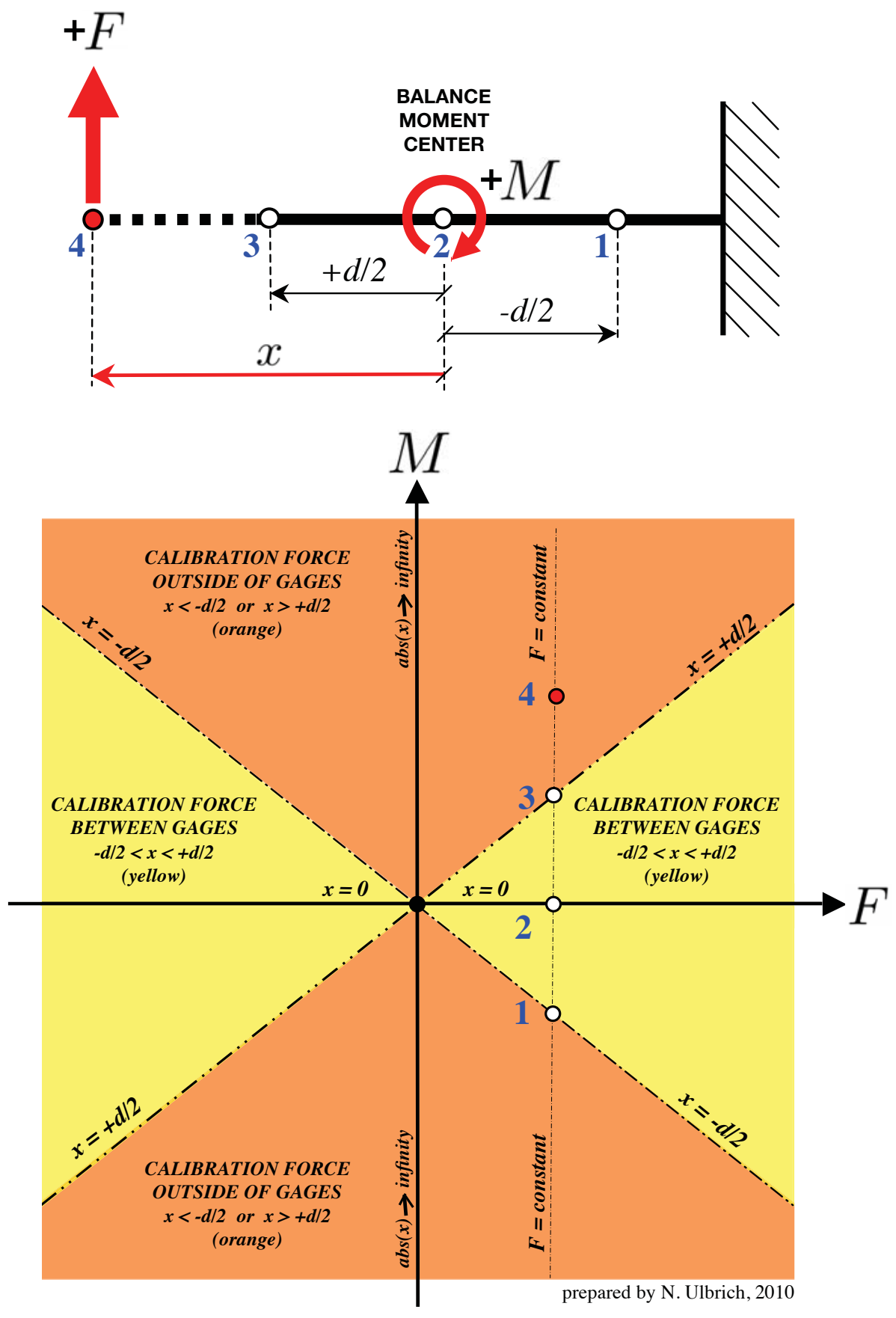

Fig. 1b Quadrant characteristics for a Direct-Read Balance. 

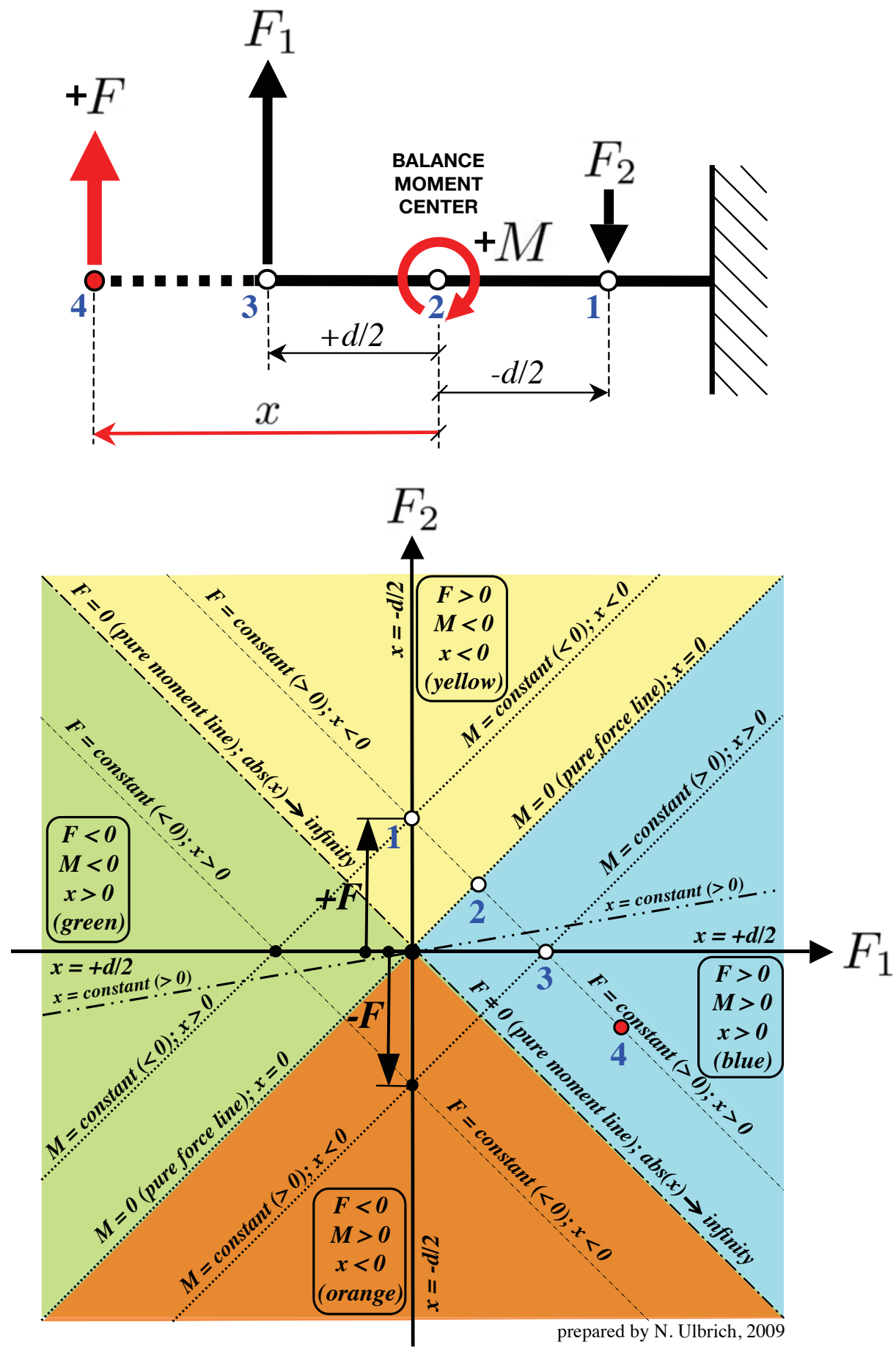

Fig. 2a Combined Load Diagram for a Force Balance. 

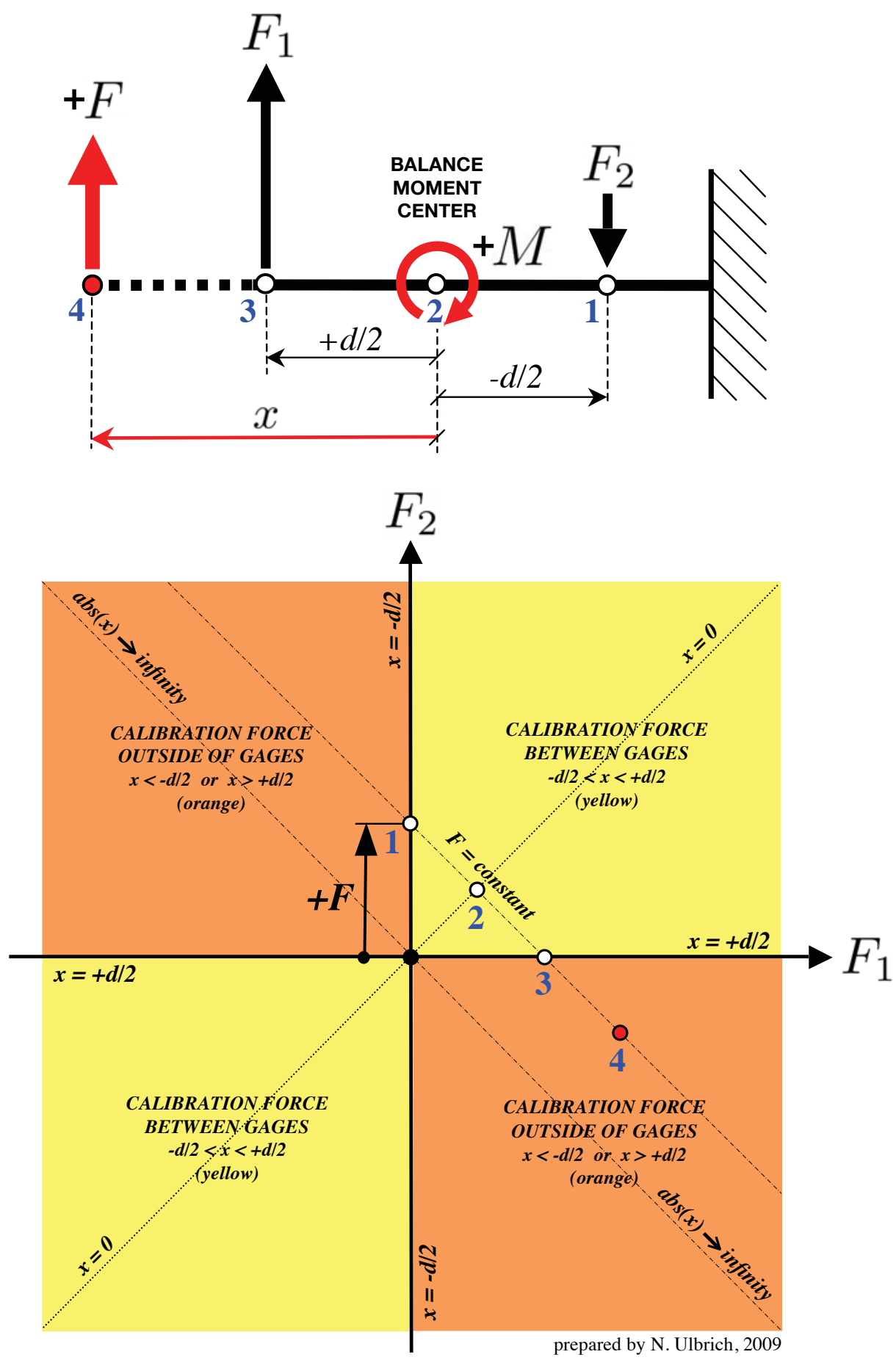

Fig. 2b Quadrant characteristics for a Force Balance. 

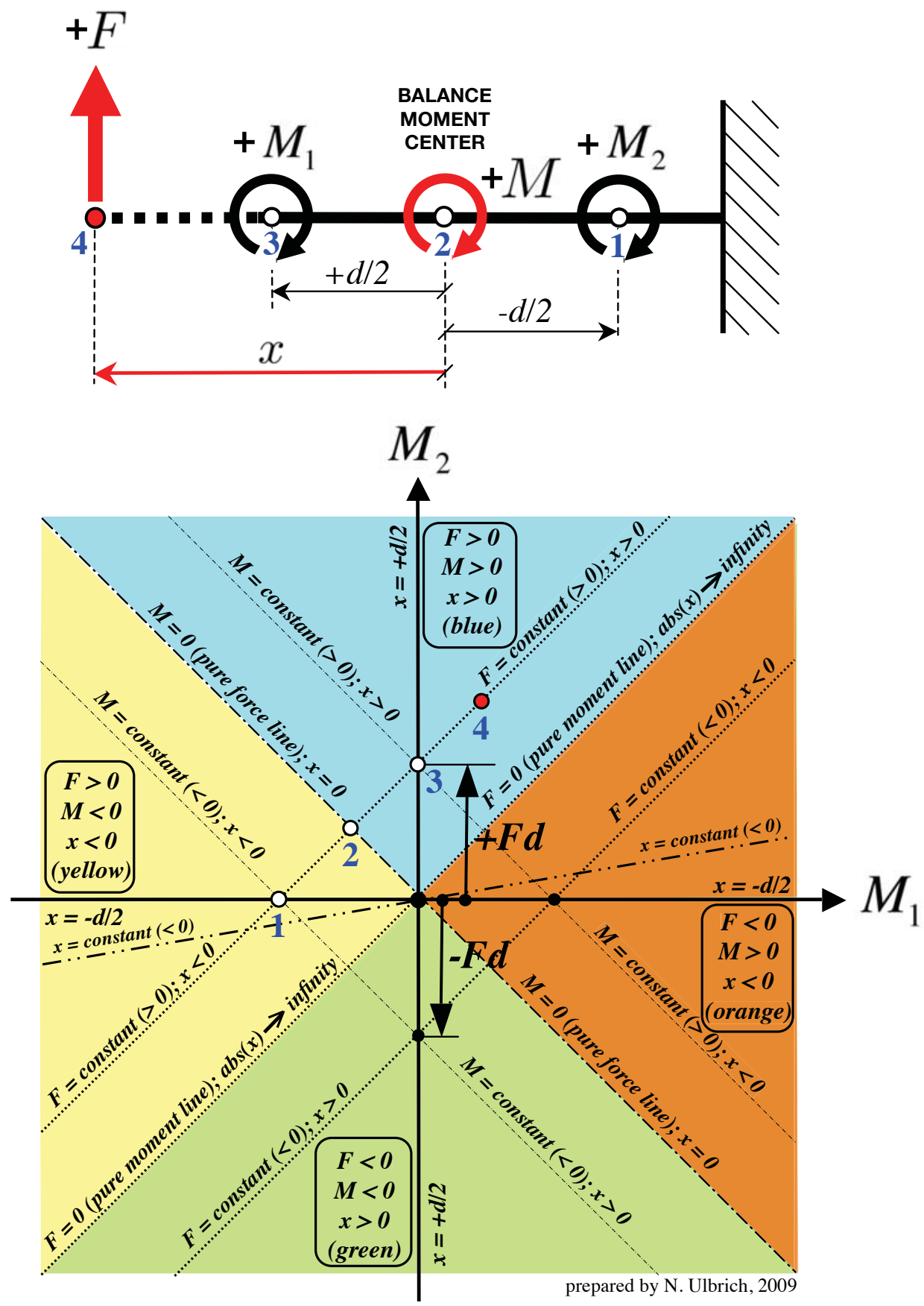

Fig. 3a Combined Load Diagram for a Moment Balance. 


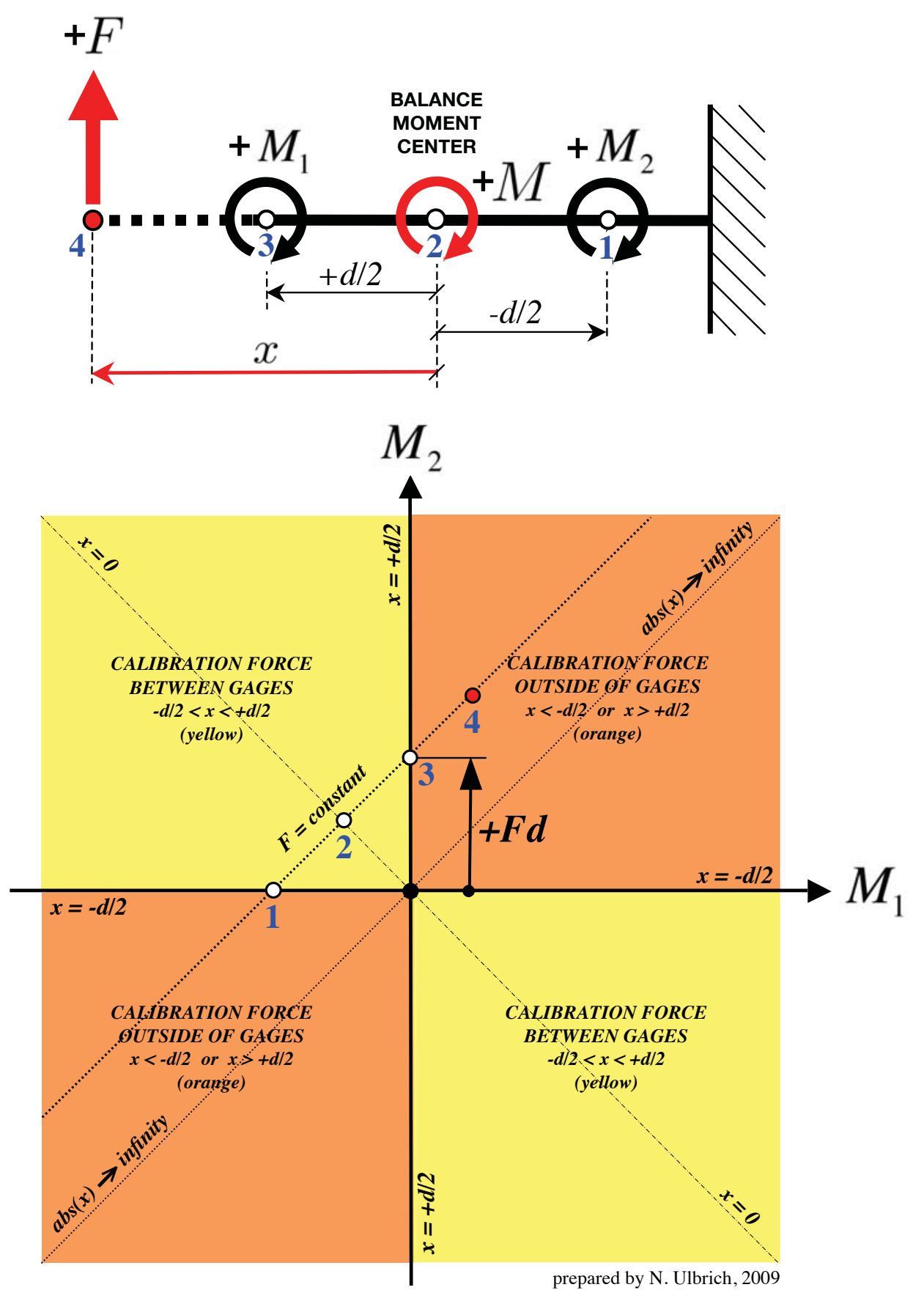

Fig. 3b Quadrant characteristics for a Moment Balance. 


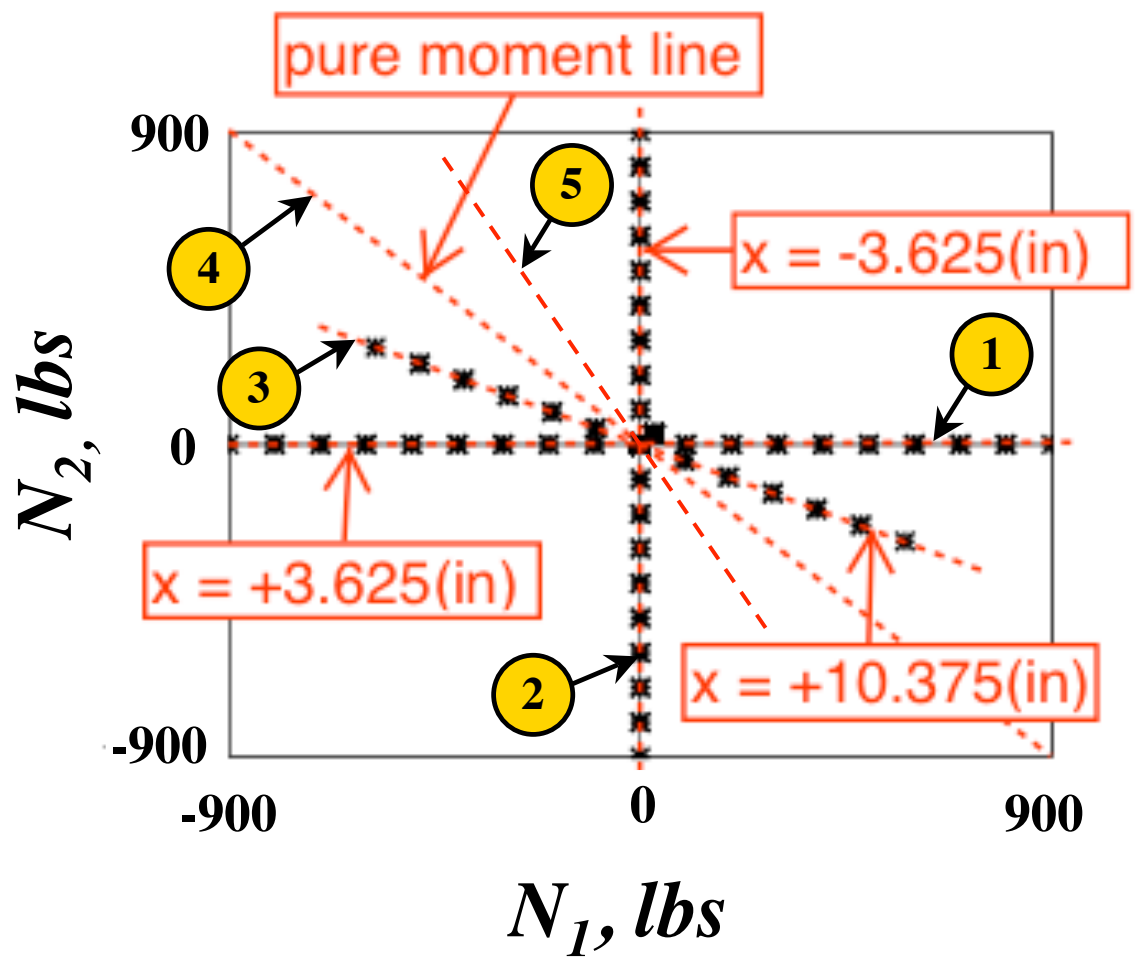

Fig. 4 Combined load plot for the normal force components of the Ames MK-III-C balance. 